\title{
Investigation of Three-Dimensional Deformation Behavior due to Long and Large Excavation in Soft Clays
}

\author{
Chunhui lou $\mathbb{D}^{1,2}$ Tangdai Xia, ${ }^{1,2}$ Nianwu Liu, ${ }^{3}$ and Xiaonan Gong ${ }^{1,2}$ \\ ${ }^{1}$ College of Civil Engineering and Architecture, Zhejiang University, Hangzhou 310058, China \\ ${ }^{2}$ Research Center of Coastal and Urban Geotechnical Engineering, Zhejiang University, Hangzhou 310058, China \\ ${ }^{3}$ Institute of Foundation and Structure Technologies, Zhejiang Sci-Tech University, Hangzhou 310018, China
}

Correspondence should be addressed to Chunhui lou; 158860104@qq.com

Received 10 December 2018; Accepted 21 March 2019; Published 30 April 2019

Academic Editor: Annan Zhou

Copyright (@ 2019 Chunhui lou et al. This is an open access article distributed under the Creative Commons Attribution License, which permits unrestricted use, distribution, and reproduction in any medium, provided the original work is properly cited.

By analyzing the extensive field data from a long and large excavation in soft clay, this study investigates the three-dimensional deformation behavior induced by excavation. Significant inhibition effects of corner on both wall deflections and ground settlements were observed and quantified. Thus, a modified function for estimating the distribution of deformation parallel to the excavation is proposed and evaluated. Further analyzing shows that the pipeline settlement can be well estimated by the modified function combining with settlements profile proposed by Hsieh and $\mathrm{Ou}$, and the reduction coefficient is about 0.8 ; the calculated maximum distortion of the pipeline can provide reliable reference. In addition, it is found that the cement-soil partition walls can also considerably reduce the wall deflections and ground settlements even after the part that is above the excavation base were removed.

\section{Introduction}

A lot of large-scale excavations are designed and constructed in an urban environment due to the demand for underground space. It is particularly important to ensure the stability and safety of excavations and the adjacent structures, especially in soft soil areas. In this regard, lots of the early work had been done to analyze the performances of deep excavations [1-5] and the mechanical properties of soft clay $[6,7]$, a number of databases have been developed, and plane strain conditions are usually assumed in designing and estimation based on these studies.

However, many researchers have found that the deformation near the corners of the excavation are smaller than that in the middle parts, which may be due to the high stiffness at the corners [8-14]. Numerical parametric analyze $[14,15]$ and centrifuge modeling $[16]$ were adopted by some researchers to simulate the three-dimensional deformation performance of the excavation and its impact on adjacent tunnels. Nevertheless, 3D numerical simulation is extremely time-consuming and difficult to calculate the deformation of large complex excavation accurately, while the centrifuge modeling has limitations in soft soil. Roboski and Finno [17] proposed an empirical function for the distribution of deformation parallel to an excavation wall, which can predict the deformation at any distance to the corner along the wall; it only requires the excavation geometry and the estimation of the maximum deformation. Nevertheless, previous researches mainly focused on the regular-size rectangular excavations, and few investigations were conducted for the large-scale excavation with a long length. Yong et al. [18] analyzed the field data of several multipropped metro station pits in Shanghai and found that corner effects of these pits were much less pronounced than the rectangular excavations. The metro station pits were usually supported by the diaphragm wall with narrow width and two station shaft on the side. Data are still rare about the large and long excavations with flexible supports.

In this study, extensive field measurement were conducted to investigate the three-dimensional performance of Lotus port excavation, which has a $470 \mathrm{~m}$ length with $38-$ $65 \mathrm{~m}$ wide. The excavation was zoned by partition walls. 
Some researches have been carried out for the performance of deep excavation with partition walls [19-21], and highstiffness diaphragm walls are used in most of the previous studies. The partition walls in this study were built by cement-soil mixing piles, which are considered as flexible walls, and the deformation behavior around the wall is summarized. In addition, some researchers have demonstrated that buried pipelines are vulnerable to damage by adjacent deep excavations [22-24]. Thus the settlement of a pipeline adjacent to this excavation was monitored and analyzed.

\section{Site Condition}

2.1. Project Overview. The site is located at the east of Lotus port river and the west of Fengtan Road in Hangzhou, China. The length of the excavation is $470 \mathrm{~m}$, and the width varied from 38 to $65 \mathrm{~m}$ in different sections. Figure 1 shows the plan view of this long and large excavation. Surrounding structures of concern included the utilities and buildings parallel to the east side of the excavation and the building (under construction) on the south side. The buildings on the east side were founded on piles. Within $20 \mathrm{~m}$ outside the excavation, there are a D300-D600 rainwater pipeline and a D1000 reinforced concrete sewage pipeline, which is shown in Figure 1.

The excavation was zoned into three strip area by two cement mixing pile partition walls consist of four-row $\varphi 850 @ 600$ cement mixing pile. Some river embankments were removed and a cofferdam of double-row steel sheet piles was designed besides the west side of Zone 1\# because of the close range between excavation and the Lotus port river. However, to reduce the project cost and the construction duration, only one row steel sheet piles were constructed. Figure 2 shows the cross section of the east side of Zone 1\#; the excavation was supported by SMW system (soil mixing wall retaining system: $\varphi 850 @ 600$ three-axis cement mixing pile with $\mathrm{H} 700 \times 300 \times 13 \times 24 @ 600 \mathrm{H}$ steel inserted) and two levels of reinforced concrete internal bracing, and the intersection was supported by using the steel column. A cement-soil grid wall was constructed for ground improvement within $6 \mathrm{~m}$ near the final excavation level in the passive zone. The east side of Zone 1\# was supported by the double-row SMW system to further control the deformation.

2.2. Geological Conditions. The site is located in a clay soil region with limnetic-plain sediments, where soft and stiff soils alternately exist. The soil properties along the depth were characterized by a series of laboratory tests and in situ tests. The typical soil stratigraphy is shown in Figure 3. The top layer is formed by backfill with a thickness of less than $2.0 \mathrm{~m}$ in general. The soft clay dominates the upper $15 \mathrm{~m}$ beneath the surface. The shallow groundwater of this site is porous phreatic water. The groundwater level is complementary to the Lotus port river and greatly affected by the fluctuation of the river water. According to the analysis of regional hydrogeological data, the annual groundwater level of the shallow groundwater is about $1.0-2.0 \mathrm{~m}$; the depth of the phreatic water level in the borehole is $1.4-3.2 \mathrm{~m}$ beneath the ground during the detailed survey.

2.3. Construction Sequence. To properly analysis the observed responses, the data should be referred to the construction activities. The site work began with the construction of cofferdam for the protection of riverway. Thereafter, the cement-soil grid wall in the passive zone and cement mixing pile partition walls were constructed when the site was cleaned. Six major construction stages are defined in Table 1: SMW installation, three stages of excavation and a quiescent stage wherein the two floor levels of the permanent structure were constructed and backfill was placed between the temporary and permanent walls, $\mathrm{H}$-shaped steels were pulled out, and the interspace was filled with cement mortar. Prior to the pulling of $\mathrm{H}$-shaped steel, the top beam and the site must be cleaned to ensure the proper function of the lifting jack and tyre crane. It is worth noting that the excessive length of the excavation would lead to an irregular excavation sequence and placement of access ramps may have caused some extraloads.

2.4. Field Observation. To monitor the horizontal displacement of the retaining walls and the deformation of the ground surface, 138 settlement survey points and 38 inclinometers were established around the excavation. The development of the strut axial forces were monitored by using 24 sets of vibrating wire stress meters. In addition, 52 settlement survey points were installed along the pipelines. Existing structures around the site are not very complicated for an excavation in urban areas. The extensive monitoring data allowed the three-dimensional response of the excavation to be captured. In this paper, only the data taken by using the inclinometer and settlement observer are analyzed.

\section{Observed Deformation Behavior}

For discussion purposes, Figure 4 presents the part of the instrumentation layout. Wherein the CX denotes the lateral displacement of the SMW retaining the walls captured by using the inclinometers, and CJ denotes the ground surface settlement behind the walls. The inclinometer CX38 is in the middle of the north side of Zone 1\#; CX4, CX9, and CX15 are in the middle of each zone; CX1, CX11, and CX18 are close to the corner of each zone; and CX5 is $53 \mathrm{~m}$ from the corner, and CX7 is at the partition wall between Zone $1 \#$ and Zone 2\#.

3.1. Lateral Wall Deflections. Figure 5 presents the typical lateral deflections of SMW walls measured at CX1, CX4, CX9, CX11, CX15, and CX18 following the completion of each excavation stages. It can be seen that the wall deflections increased with excavation and concavely developed. The depth of the maximum deflections mostly falls at the final excavation level or slightly above it. 


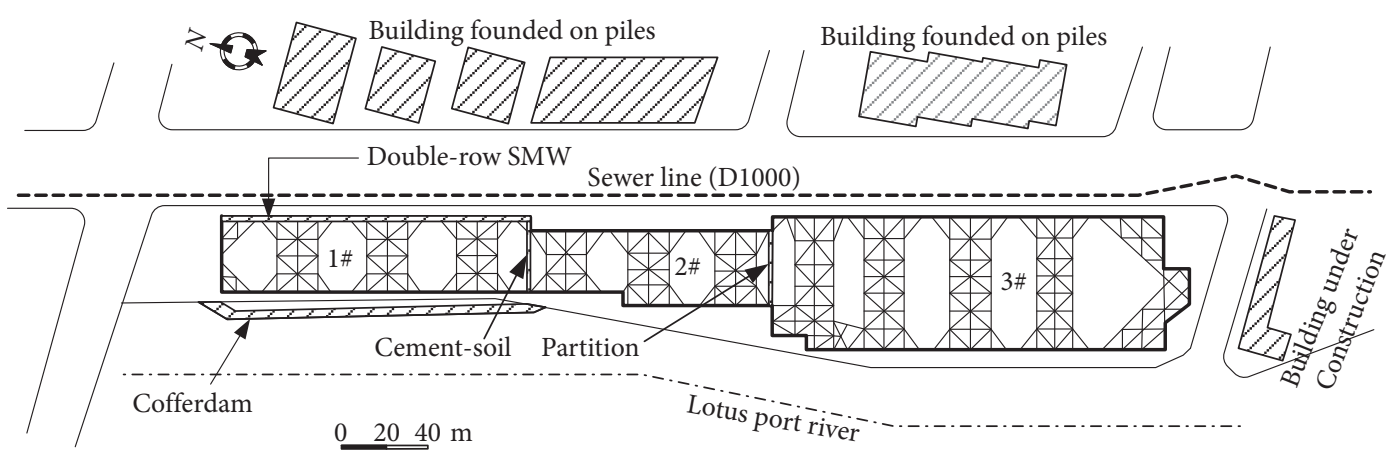

Figure 1: Plan view of the site.

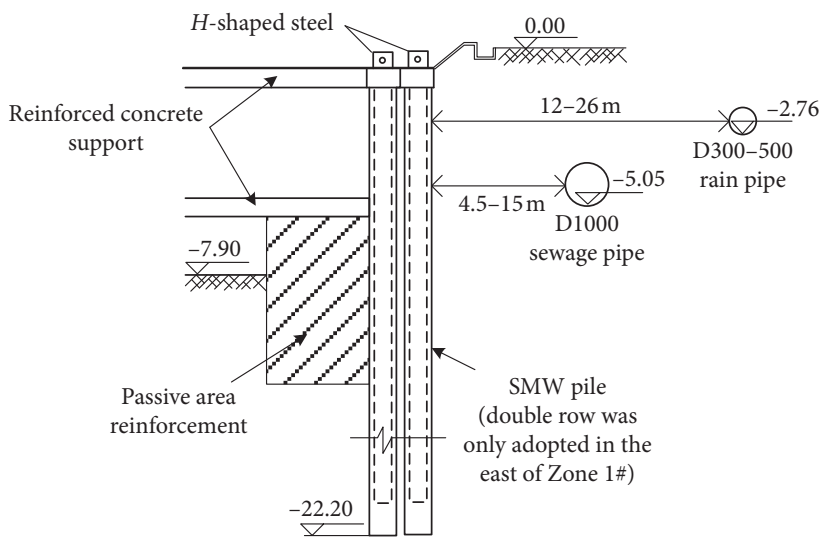

Figure 2: Typical cross section.

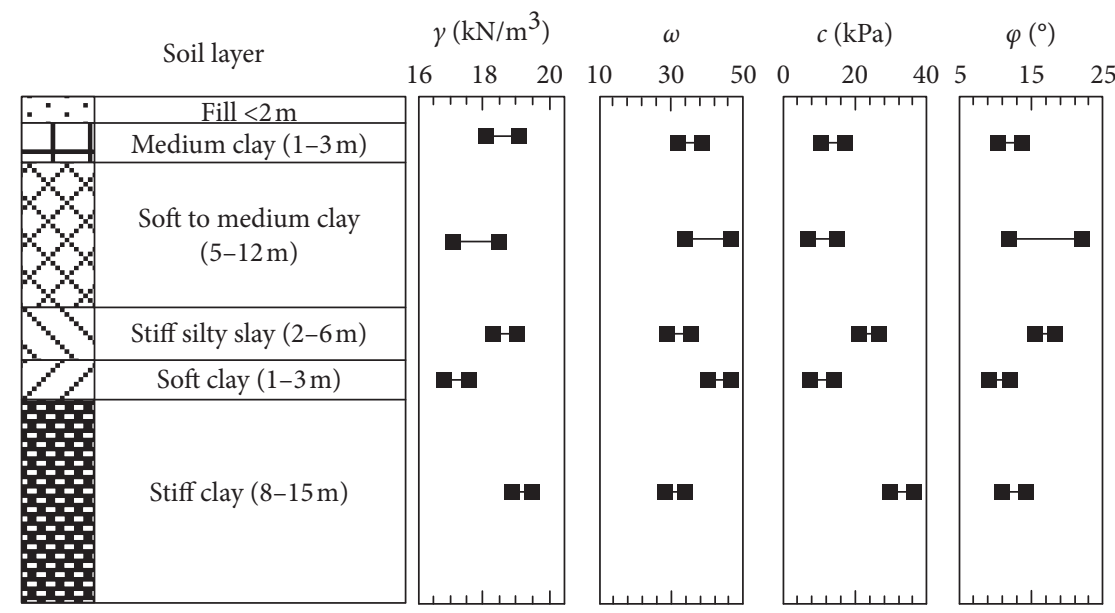

Figure 3: Subsurface conditions at the Lotus port excavation.

TABle 1: Summary of construction activities.

\begin{tabular}{lc}
\hline Stage number & Activity \\
\hline 1 & Reinforce the passive zone, install SMW, and insert H-shaped steel \\
2 & Excavate to $-1.8 \mathrm{~m}$ and pour the first layer of concrete internal bracing \\
3 & Excavate to $-6 \mathrm{~m}$ and pour the second layer of concrete internal bracing \\
4 & Excavate to $-7.9 \mathrm{~m}$ and pour grade beams \\
5 & Construct basement walls, pour slab, and demolish the second internal bracing and backfill \\
6 & Pull out the H-shaped steel, fill the interspace, and demolish the first internal bracing \\
\hline
\end{tabular}




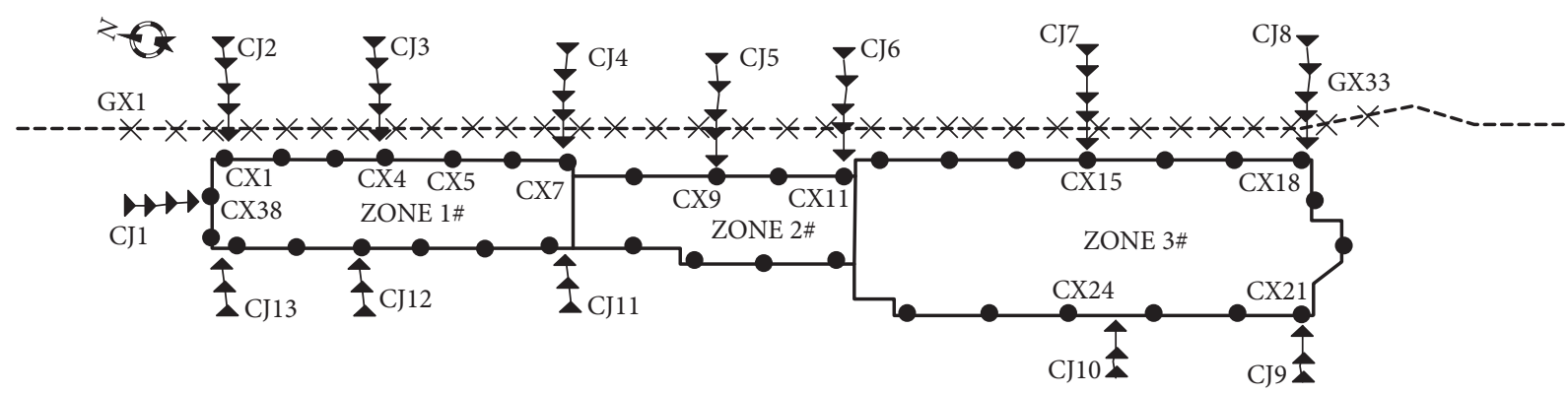

- CX: inclinometer

- CJ: ground settlement

$X$ GX: pipeline monitoring point

Figure 4: Plan view of monitoring points. Note: only part of settlement point is shown.

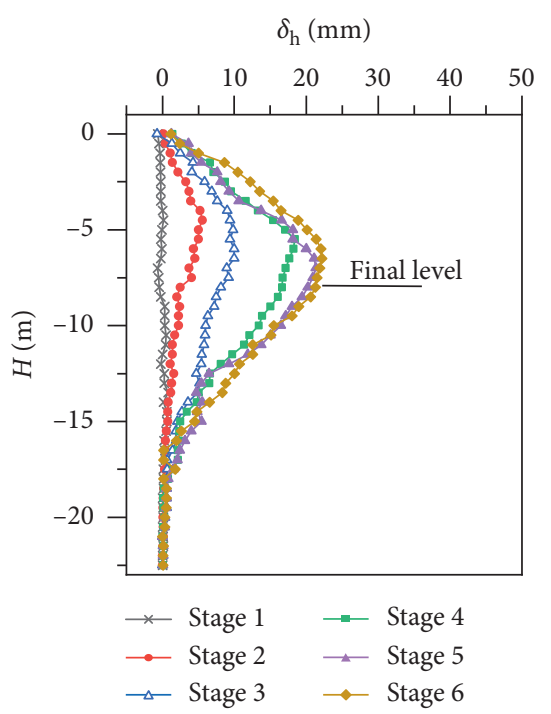

(a)

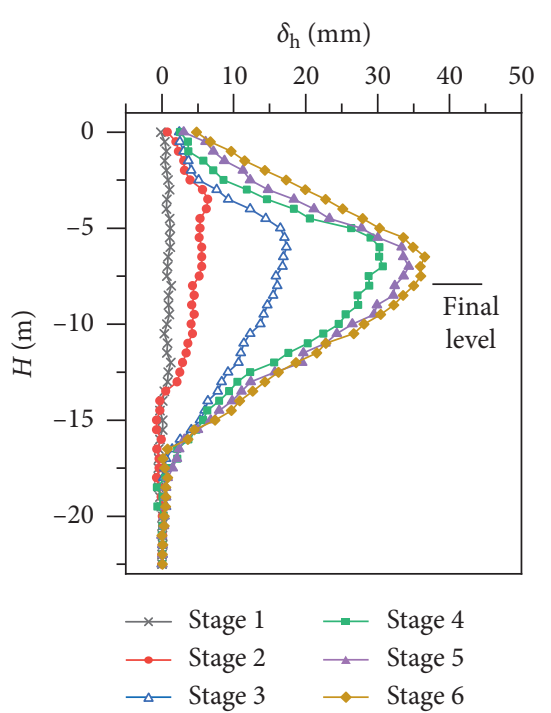

(d)

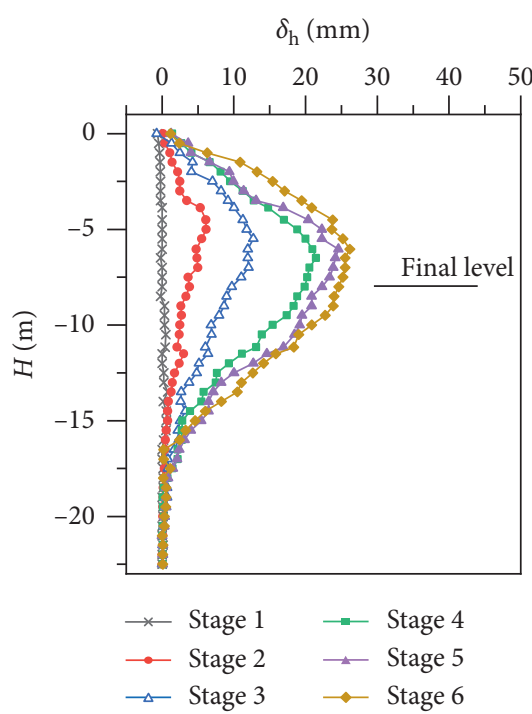

(b)

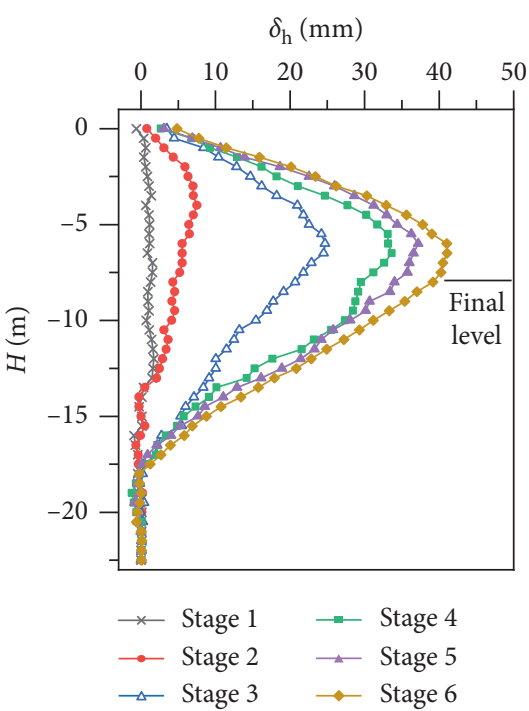

(e)

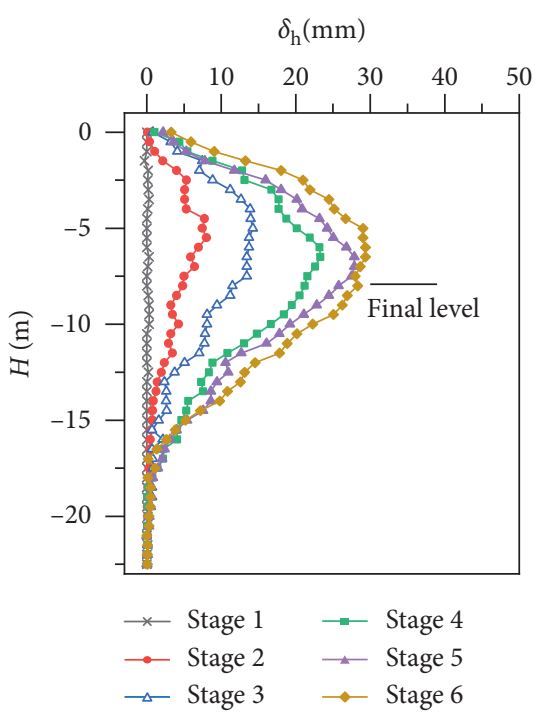

(c)

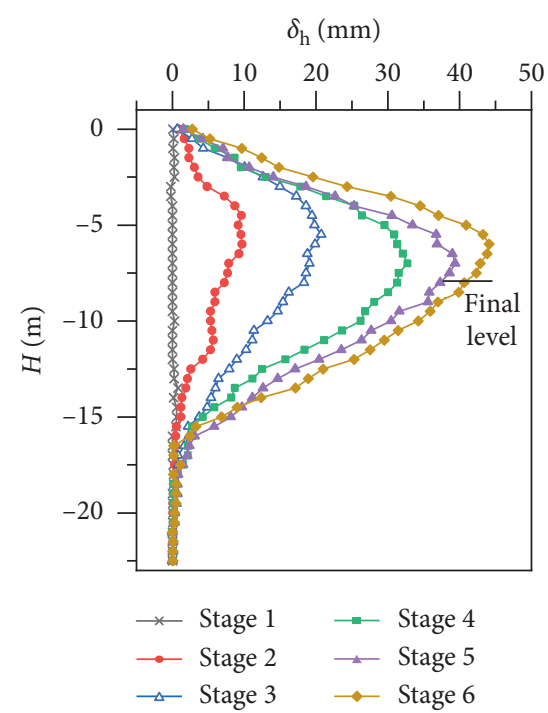

(f)

Figure 5: Wall deflections measured at (a) CX1, (b) CX11, (c) CX18, (d) CX4, (e) CX9, and (f) CX15. 
By observing the data of CX9, the development between stage 2 and stage 3 was faster than that of CX4. The possible reason is that when Zone 2\# was excavated from level 1 to level 2, the soil transportation stopped about 10 days due to the government's transportation control while the excavation was still continuing. A lot of soils were stacked on the ground outside the east of Zone 2\#, which may have caused additional loads to the ground and increased the wall deflections.

Comparing the data in first row of Figure 5 with the second row, the wall deflections near the corner are considerably smaller than that in the middle of each zone at every stage. The maximum wall deflections at CX1, CX11, and CX18 were smaller than those of CX4, CX9, and CX15 by $39 \%, 36 \%$, and $34 \%$. Figure 6 shows the wall deflections at final stage versus the distance to the corner on both sides of Zone $1 \#$. As can be seen, there is a significant trend of increase from corner towards the midspan, and other zones also have the similar characteristics. Results suggest that the wall deflections around the corners are significantly inhibited comparing to the deflections near the midspan at all three zones; it is inaccurate to practice plane strain analysis in this project, especially for the areas near the corner.

Figure 7 shows the comparison of maximum wall deflections at CX38, CX1, CX4, CX5, and CX9. Comparing CX9 (i.e., middle of Zone 2\#) with CX4 (i.e., middle of Zone $1 \#)$, it can be seen that the maximum deflection at CX9 was about $12 \%$ larger than CX4. Except the soil stacking problem mentioned before, the double-row SMW was adopted on the east side of Zone 1\#, suggesting that the double-row SMW had extrasupport effect for the excavation. Combined with other data collected, the constraint effect was about $10-20 \%$ better than the single-row SMW system.

Figure 7 also shows that the maximum wall deflections at CX5 are quite close to CX4, implying that the PSR (plane strain ratio) at CX5 is close to 1.0. The concept of PSR was proposed by Ou et al. [10] to describe deflection behavior of a wall section. It was defined as $\mathrm{PSR}=\delta_{\mathrm{hm}, \mathrm{d}} / \delta_{\mathrm{hm}, \mathrm{ps}}$, where $\delta_{\mathrm{hm}, \mathrm{d}}$ is the maximum wall deflection at a certain section of the wall and $\delta_{\mathrm{hm} \text {,ps }}$ is the maximum wall deflection under plane strain condition. PSR equals to 1.0 which represents that the sections are under the plane strain condition since the distance of CX5 from the partition wall corner was $53 \mathrm{~m}$ and it was about $20 \mathrm{~m}$ away from the center. The results may suggest that after a certain distance away from the corner, the wall deflections are no longer sensitive to the total length of the excavation; the inhibition effect of the corner will be invalid.

In addition, the wall deflection at CX38 on the north side is smaller than that of CX4 and CX9. Since the total length of the north wall is $38 \mathrm{~m}$, the corner may still have inhibition effect on the middle part of the wall, and the wall at CX38 had not reached the plain strain condition yet. The detailed method to evaluate the corner effect is summarized in Section 4.

3.2. Maximum Wall Deflections. Figure 8 shows the relationship between $\delta_{\mathrm{h}}$ and $H_{\mathrm{e}}$; based on the previous

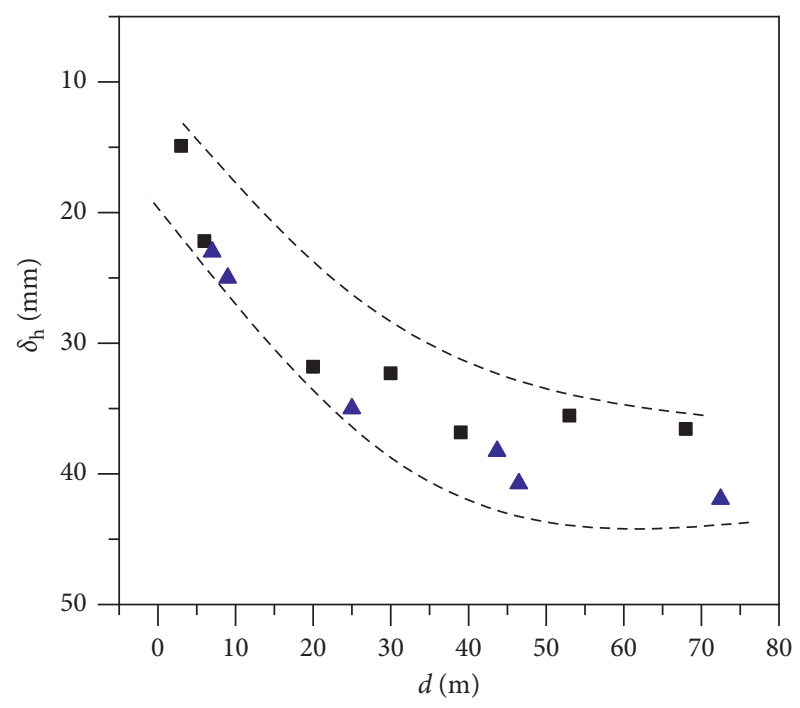

Zone 1\#

- East side

A West side

FIgURE 6: Wall deflections versus the distance to the corner in Zone 1\#.

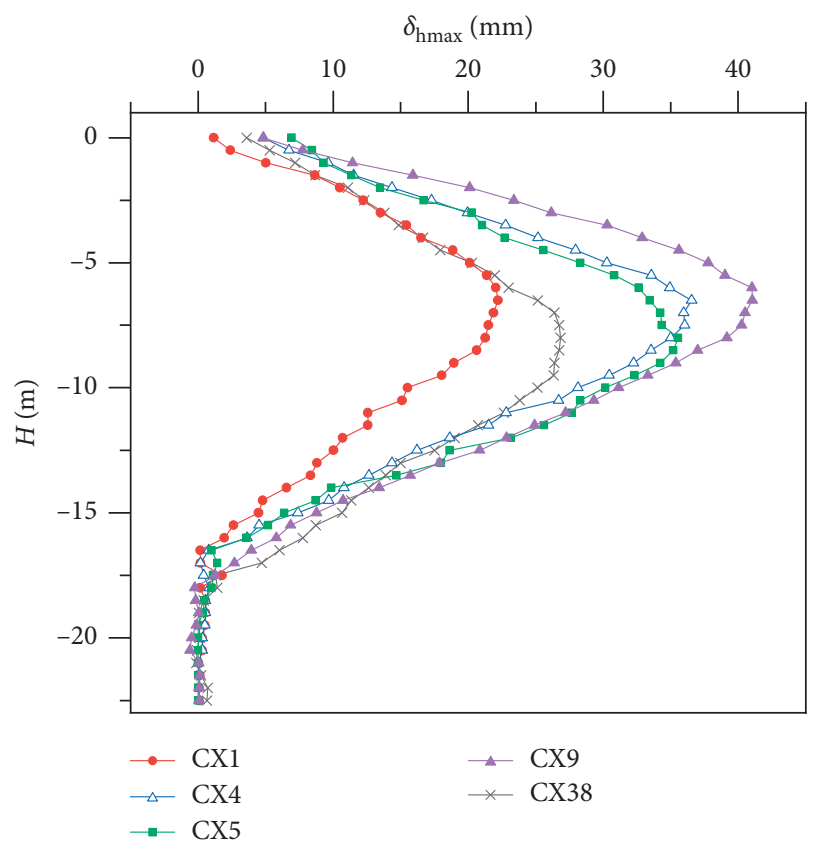

FIgURE 7: Comparison of maximum wall deflections.

literatures, some empirical relationships between $\delta_{\mathrm{h}}$ and $H_{\mathrm{e}}$ have been summarized. Peck [1] suggested the limiting line, $\delta_{\mathrm{h}}=1 \% H_{\mathrm{e}}$, based on the excavations data in soft clays supported by sheet piles or soldier piles. It was observed that measured $\delta_{\mathrm{h}}$ in this case was significantly smaller than the profile proposed by Peck. Most of the data points fell between $0.2 \%$ and $0.6 \% H_{\mathrm{e}}$ which was close to the range proposed by Kung et al. [3] for excavations in soft to medium clay but larger than that of Clough [2] for excavations in stiff clay. 


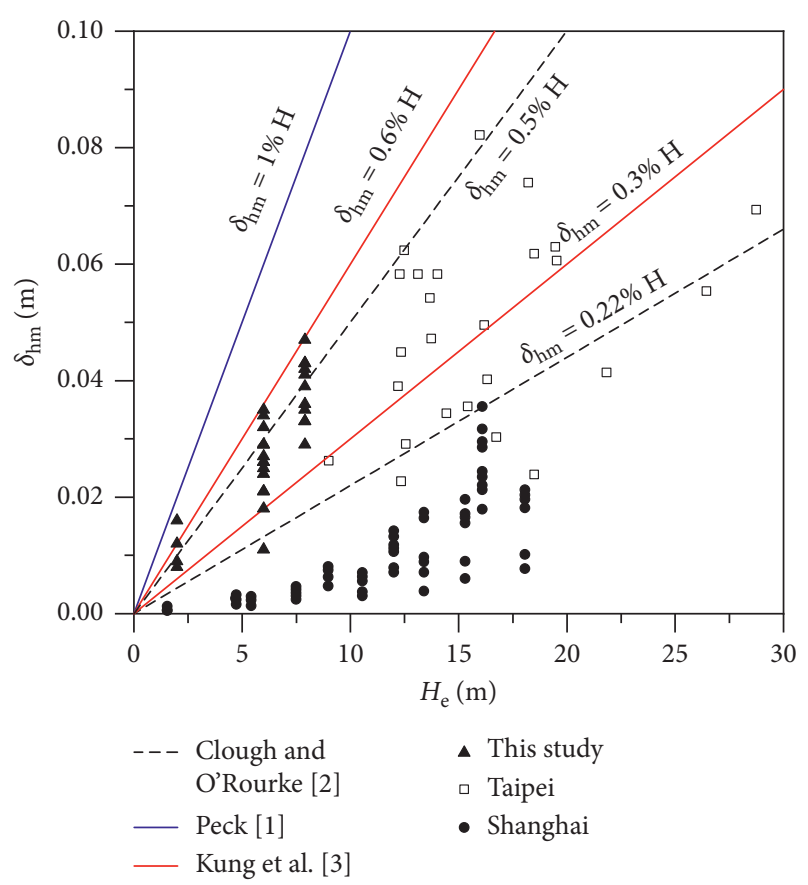

FIGURE 8: Relationship between the maximum wall deflections and excavation depths.

By comparing with excavations at Taipei (Ou et al. [4, 5], Hsieh and Ou [25], and Kung et al. [3]) and Shanghai (Tan et al. [26]), results imply that although the excavation depths in Taipei were much deeper, the measured $\delta_{\mathrm{h}}$ was in the close range with this study or slightly smaller. Moreover, the measured $\delta_{\mathrm{h}}$ in the Shanghai case was significantly smaller. This is probably a consequence of two main reasons: firstly, the diaphragm walls that adopted in those cases had considerable better support than the SMW retaining system; secondly, the long length and large scale of this excavation leaded to the long duration of wall exposure. Although the subway excavation in Shanghai also had a large length, the width was only $20 \mathrm{~m}$. It suggests that the ratio of width to length of excavation may also affect the wall deflections. In addition, the passive zone around the excavation base in this case was reinforced with the cement-soil grid wall, but it was usually difficult to ensure the construction quality. Consequently, it had a good effect in blocking the underground water but limited effect in reducing wall deflections.

3.3. Ground Surface Settlement. Figure 9 shows the measured ground surface settlements perpendicular to the excavation versus distance behind the walls, and two commonly used nondimensional ground settlement profiles (Clough [2] and Hsieh and $\mathrm{Ou}$ [25]) are also shown for comparison. As can be seen, the trend of the normalized settlement is quite similar to the profile of Hsieh and $\mathrm{Ou}$.

As shown in Figure 10, the settlements at CJ5 and CJ7 are larger than $\mathrm{CJ} 3$, suggesting that the double-row SMW wall also played a certain role in limiting the ground settlement. Only three rows of settlement measurement points were arranged on the west side of Zone 1\# due to the close distance

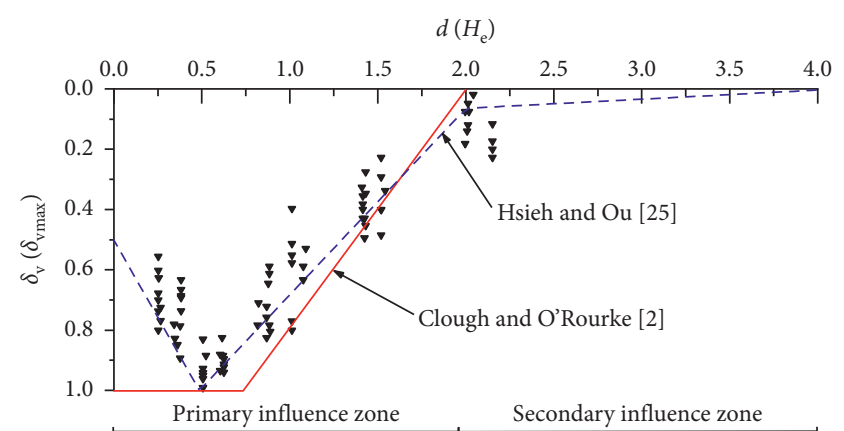

FIgURE 9: Observed settlement profiles versus empirical methods.

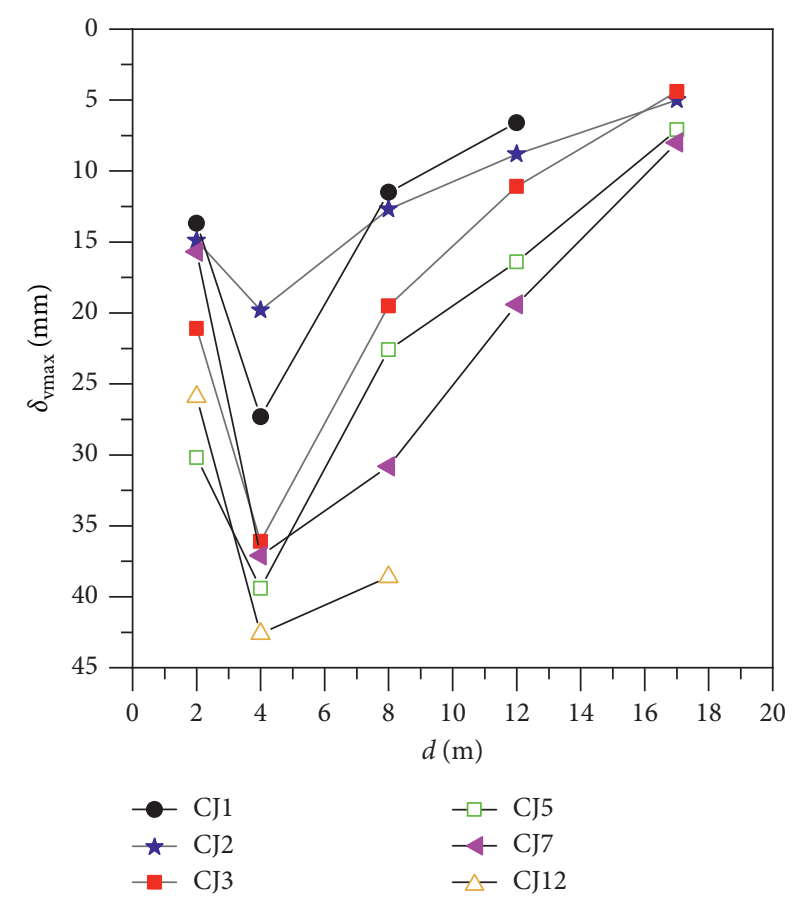

FIGURE 10: Comparison of maximum ground surface settlement profiles.

between river and retaining wall, and the settlement of CJ12 is larger than CJ3 and shows a rapid development. This may be due to the deformation of the cofferdam. During the excavation, the new steel sheet pile cofferdam also had a certain displacement towards the direction to the Lotus port river, which may have caused part of the ground surface settlements. In addition, as shown in Figure 11, similar to the distribution of wall deflections, the maximum ground settlements outside the corner of each zone are about $43 \%$ smaller than those of the middle section in average, indicating that the ground surface settlements near the corners are also significantly inhibited.

3.4. Restrain Effect of the Partition Wall. Figure 12 shows the development of wall deflections and ground surface settlements on the east side of the partition wall between Zone 1\# and Zone 2\#. It should be noted that the stage division shown here is different from the construction process of the 


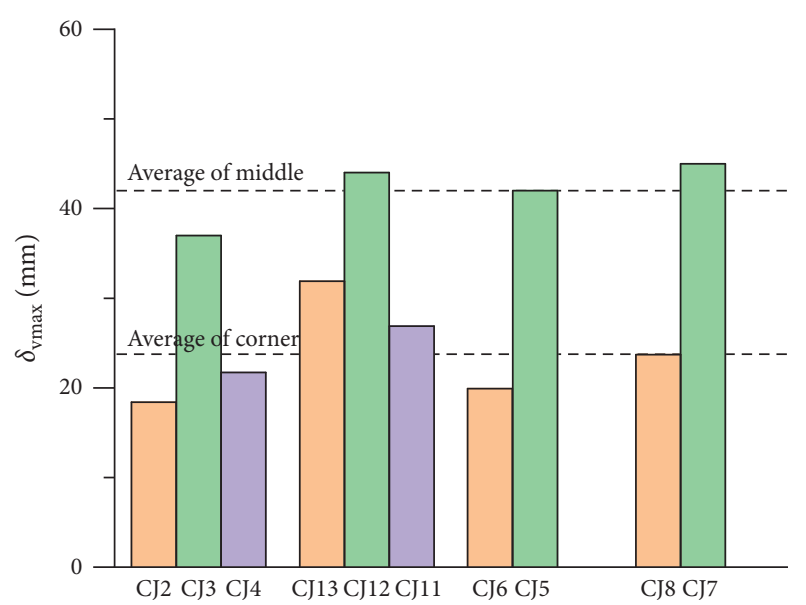

Figure 11: Comparison of maximum ground surface settlement values.

standard sections but based on five stages as shown in Table 2.

The results suggest that in the excavation stage of Zone $1 \#$, the wall deflection and ground surface settlement at the partition wall had been well restricted. When the excavation of Zone 2\# began and part of partition wall was removed, the lateral displacement had a certain development, but the shapes of the curve are different from other positions, and the depth of the maximum deformation was also significantly higher than the excavation base. After the excavation completed in Zone 2\#, the maximum lateral displacement and settlement are significantly smaller than that in the central part of Zone $1 \#$ or Zone 2\#.

The principle of restrain effect of the partition wall could be as follows: when the partition wall was intact, the wall directly supported the retaining wall in the vertical direction, which almost completely limited the wall deflections. After the wall above the excavation base was removed, due to the certain insertion depth of the cement-soil wall, the soils below the excavation base were reinforced, and the basal heaves were limited to a certain extent, which reduced the wall deflections and ground settlements.

\section{Modified ERFC Fit Based on Survey Data from the Lotus Port Excavation}

4.1. Origin Fit Curve. Roboski and Finno [17] found that the distribution of lateral wall movement and ground movement in a direction parallel to an excavation could be simulated as an empirical equation as follows:

$$
\delta(x)=\delta_{\max }\left[1-\frac{1}{2} \operatorname{erfc}\left(\frac{2.8(x-A)}{0.5 L-A}\right)\right],
$$

where $\delta_{\max }$ is the maximum deformation and $A$ is the distance from the inflection point of the curve to the excavation corner as shown in Figure 13. A could be calculated by a fit curve that describes the relationship of $A$ normalized by one-half the wall length and $H_{\mathrm{e}}$ normalized by wall length using the data from the Robert $\mathrm{H}$. Lurie Medical Center project. The expression of fit curve is

$$
\frac{2 A}{L}=-0.069 \ln \left(\frac{H_{\mathrm{e}}}{L}\right)-0.03 .
$$

In order to explain the process of predicting the deformation distribution using equation (1), a flow chart was made as shown in Figure 14.

4.2. Modification of ERFC Fit Curve. However, the excavation in the Robert $\mathrm{H}$. Lurie Medical Center project is not a long shaped or oversized excavation, and the $H / L$ ratio is mostly concentrated at $0.1-0.25$. At present, oversized excavations or extremely long shaped excavations are becoming more and more common, such as the excavation in this study. The length of the long side of excavation is still over $100 \mathrm{~m}$ after partitioning. Owing to the intensive monitoring in this project, a large number of data between $H / L$ 0.05-0.21 were collected. During the research, it was found that the calculated $A$ from measured data have a certain discrepancy from the original fit curve in some intervals. Thus the calculated $A$ values are summarized in Figure 15.

The data of the Robert H. Lurie Medical Center project and the origin fit curve are presented for comparing. As can be seen, when $H / L$ is lower than 0.13 , the $A$ values in this project demonstrate a different trend from the origin fit curve. Results indicate that the original prediction curve may have certain error for an oversized or long excavation, which has lower $H / L$. By trial and error, another curve was fit to modify the relationship between the $2 \mathrm{~A} / \mathrm{L}$ and $H / L$ in the range that $H / L$ is less than 0.13 which is also shown in Figure 13, and the expression of the new curve is

$$
\begin{aligned}
\frac{2 A}{L} & =-0.78\left(\frac{H_{\mathrm{e}}}{L}\right)^{2}+0.12, \\
A & =\frac{-0.39 H_{\mathrm{e}}^{2}}{L}+0.06 L .
\end{aligned}
$$

Excavation is a complicated process. There are many reasons that may lead to the disparity between the data and the original fitting curve such as the shape of the excavation, different support system, influence of the external environment, and different soil conditions. In addition, some theories may be related such as the PSR concept mentioned in Section 3.1. That theory indicates that higher PSR means the corner has less effects on the section. This PSR value is also related to many factors, such as soil condition, support system form, aspect ratio of excavation, and so on. It is also found that when $L / H$ is larger than a certain value, the central part of excavation will be in a plane strain condition. Oversized or long excavations usually have large $L / H$. Therefore, after a certain distance from the corner, the excavation section will be in the plane strain condition. In contrast, it can be seen from the origin curve that when $H$ is invariant, $A$ would keep increasing along with the increase of $L$ and the rate of increase will get bigger. However, the influence of $L$ on the corner effect should be less pronounced 


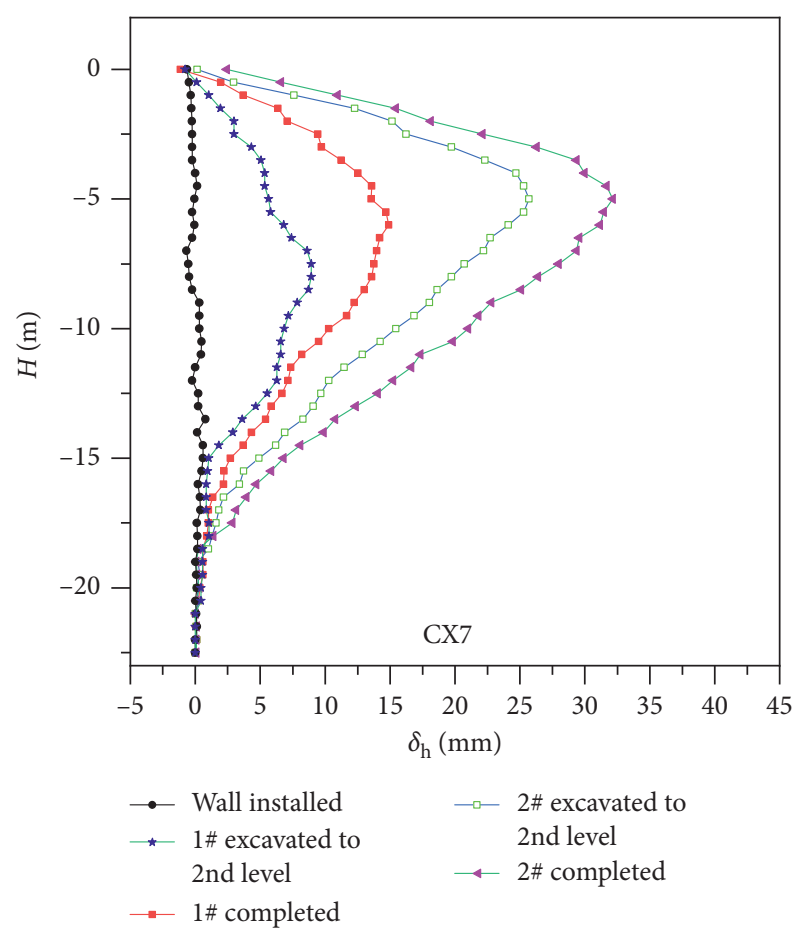

(a)

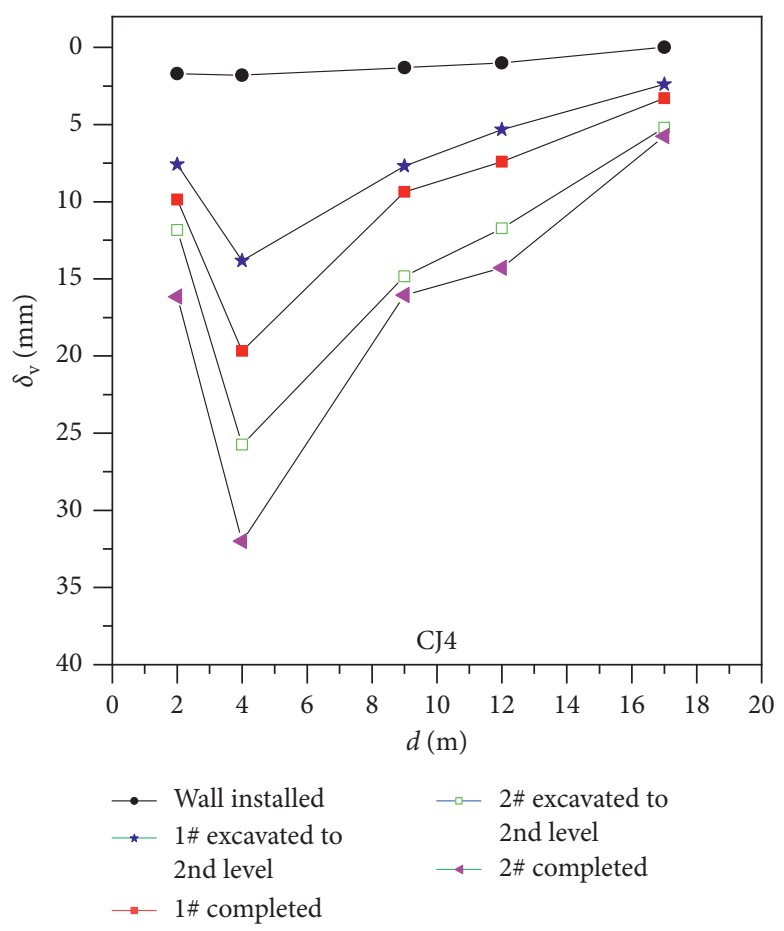

(b)

Figure 12: Observed settlement and wall deflections at partition wall. (a) CX7 and (b) CJ4.

TABLe 2: Summary of construction activities.

\begin{tabular}{lc}
$\begin{array}{l}\text { Stage } \\
\text { number }\end{array}$ & Activity \\
\hline 1 & Wall was installed \\
3 & $\begin{array}{c}\text { Zone 1\# was excavated to the second support } \\
\text { Zone 1\# was excavated to the base }\end{array}$ \\
4 & $\begin{array}{c}\text { Zone 2\# was excavated to the second support, and } \\
\text { the partition wall was removed accordingly } \\
\text { Zone 2\# was excavated to the base, and the } \\
\text { partition wall above the base was removed }\end{array}$ \\
\hline
\end{tabular}

when $L / H$ reaches a certain value according to the research of $\mathrm{Ou}$ et al. [10]. This may also explain the disparity between the data and the original fit curve.

Substituting equation (4) into equation (1) yields new expressions for the distribution of deformation parallel to the excavation:

$$
\delta_{\mathrm{vmi}}=\delta_{\mathrm{vm}}\left[1-\frac{1}{2} \operatorname{erfc}\left(\frac{2.8\left(x+0.39 H_{\mathrm{e}}^{2}\right) /(L-0.06 L)}{0.44 L+0.39 H_{\mathrm{e}}^{2} / L}\right)\right] .
$$

In addition, the corresponding maximum distortion of deformation can be expressed as

$$
\text { maximum distortion }=\frac{2.8 \delta_{\max }}{\left(0.44 L+0.39 H_{\mathrm{e}}^{2} / L\right) \sqrt{\pi}} .
$$

The maximum distortion occurs at the inflection point; it can be used to estimate the shearing effect on surrounding buildings or pipelines due to the excavation.
Owing to these two well-documented excavation data, a more conservative range of the position of inflection point (value A) is proposed; it can be enveloped by the following two curves as shown in Figure 15:

$$
\begin{cases}A_{\text {imax }}=-0.2 H_{\mathrm{i}}+0.11 L_{\mathrm{i}}, & \text { upper bound, } \\ A_{\text {imin }}=-0.2 H_{\mathrm{i}}+0.06 L_{\mathrm{i}}, & \text { lower bound. }\end{cases}
$$

This envelope can be used as a reasonable reference for determining the high-risk area near the corner of the excavation that may be damaged by the distortion.

4.3. Evaluation of the Modified Fit Curve. Roboski and Finno [17] suggested the usage of the standard deviation of the residual error to evaluate the "goodness of fit" as follows:

$$
\text { error }=\frac{\sqrt{(1 / n-1) \sum_{i=1}^{n}\left(\delta_{\text {obsi }}-\delta_{\text {erfci }}\right)^{2}}}{\delta_{\max }} \times 100,
$$

where $\delta_{\text {obsi }}$ is the observed data and $\delta_{\text {erfci }}$ is the calculated data from the fit curve. As shown in Figure 16, all the error of calculated data is less than $6 \%$, suggesting that the modified fit curve is a reasonable one. The origin fit curve is also shown in Figure 16, and it can be seen that when $L$ is small $(L=38 \mathrm{~m})$, the two fit curves are quite close and all fit the observed data; however, when $L$ is large, the predicted values of the original curve underestimates the deformation compared to observed data. $\Delta A_{\mathrm{i}}$ that is defined as the difference between the $A$ of original curve and modified curve is about $8 \mathrm{~m}$ in Zone $3 \#$. 


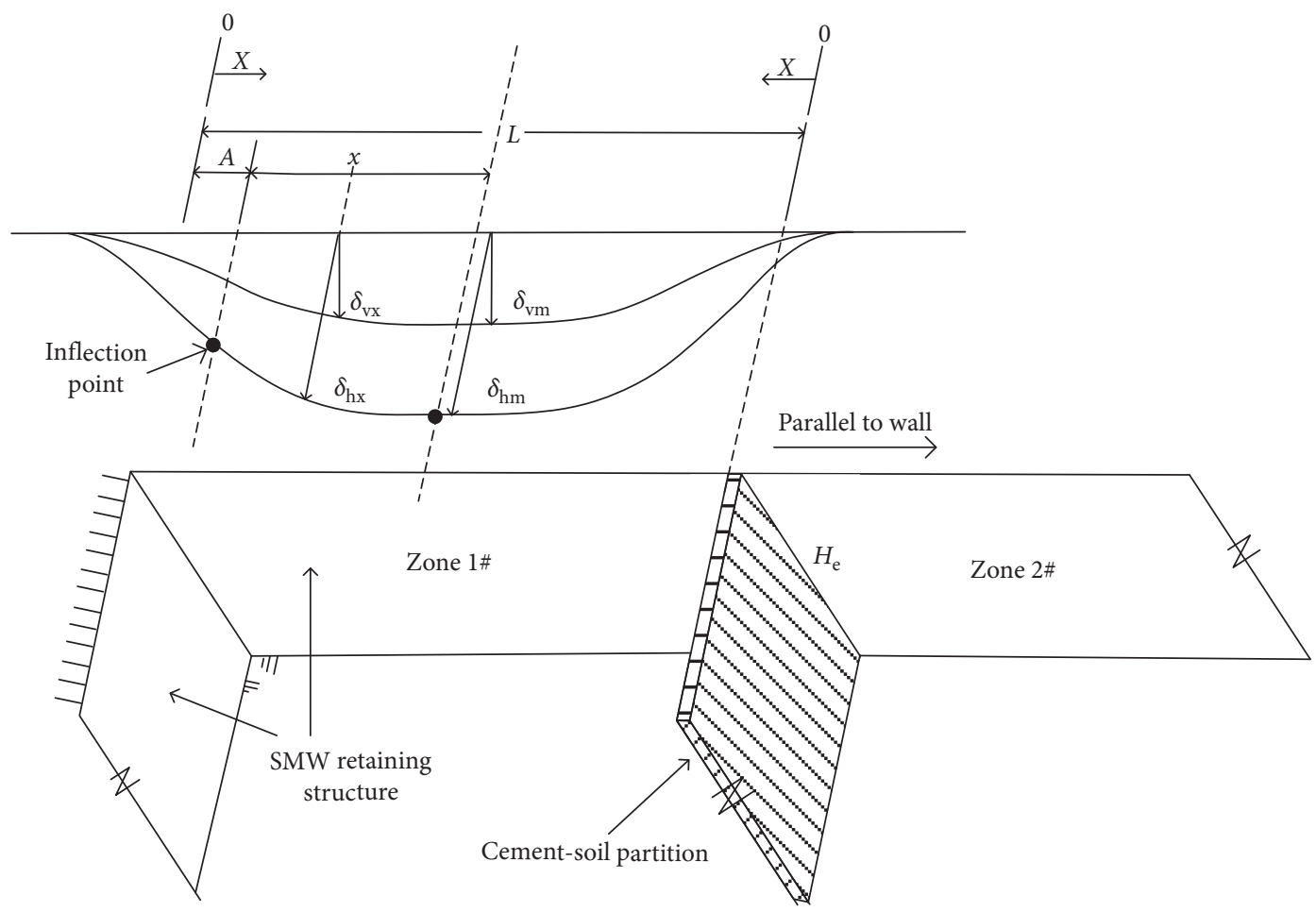

FIGURE 13: ERFC fitting schematic and notation of parameters. $\delta_{\mathrm{vx}}$ : ground surface settlement. $\delta_{\mathrm{vm}}$ : maximum ground surface settlement. $\delta_{\mathrm{hx}}$ : lateral wall deflection. $\delta_{\mathrm{hm}}$ : maximum lateral wall deflection. $H_{\mathrm{e}}$ : final excavation depth. $L$ : excavation length. $A$ : distance from the corner to the inflection point.

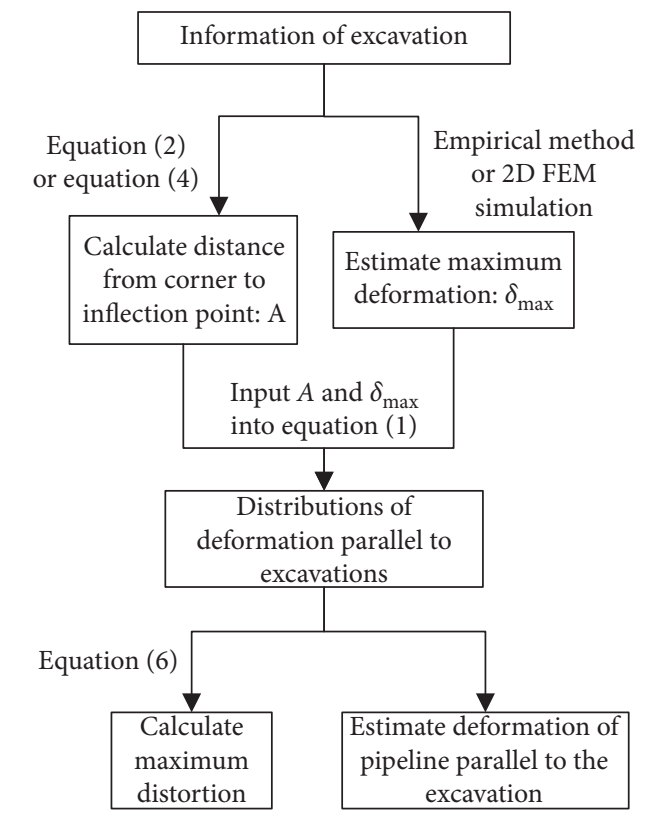

Figure 14: Flow chart for the process of predicting.

\section{Prediction of Pipeline Settlement along the East Wall}

Prediction of deformation parallel to the excavation is particularly useful for estimating the deformation of the pipeline outside the excavation. When the corner of the

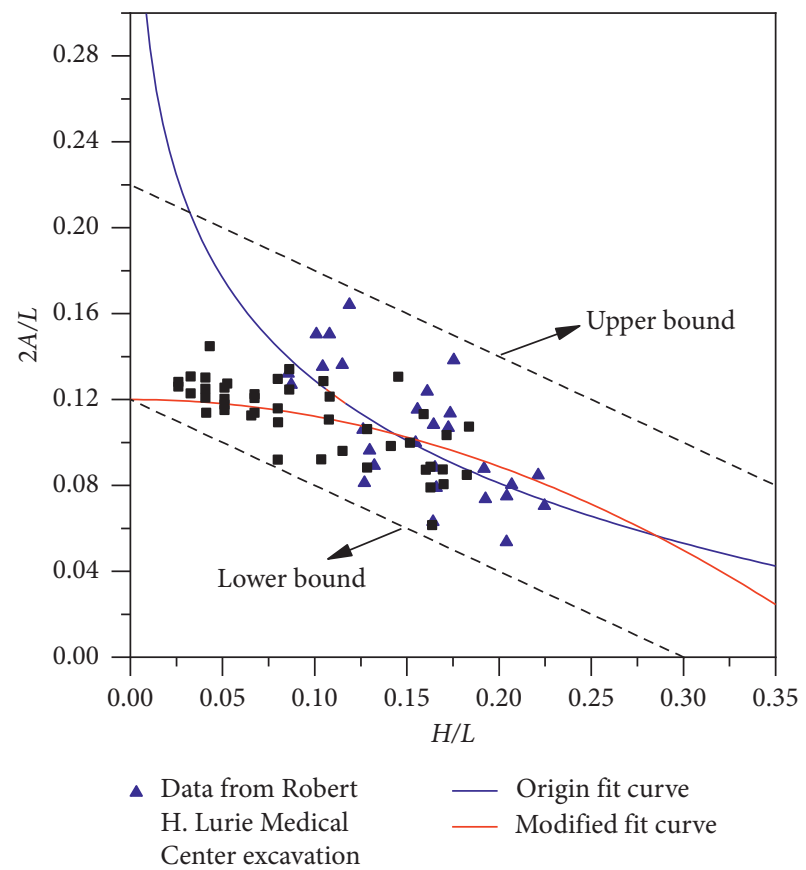

- Data from this site

Figure 15: $2 A / L$ versus $H_{\mathrm{e}} / L$ and fit curve.

excavation limits the ground surface settlement and lateral displacement, the deformation of the underground pipeline at the same position may also be suppressed. The inhibition effect will cause the differential deformation of pipeline, which may result in shear damage. 


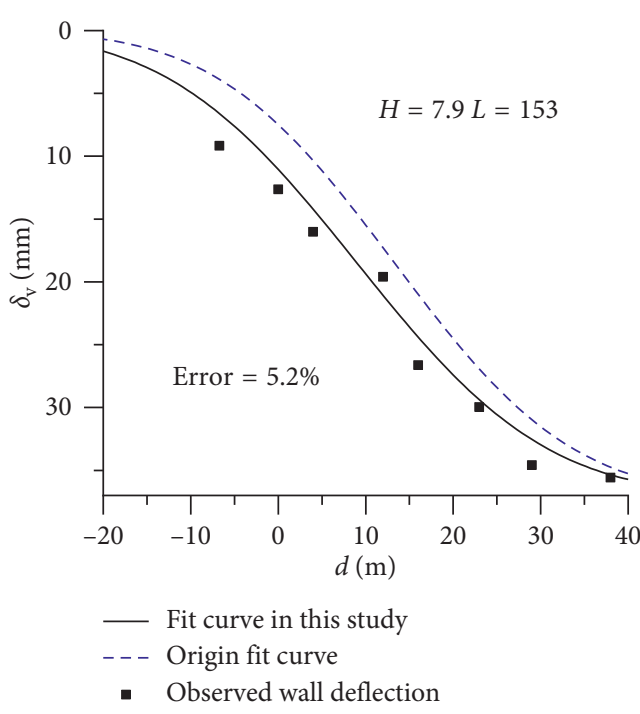

(a)

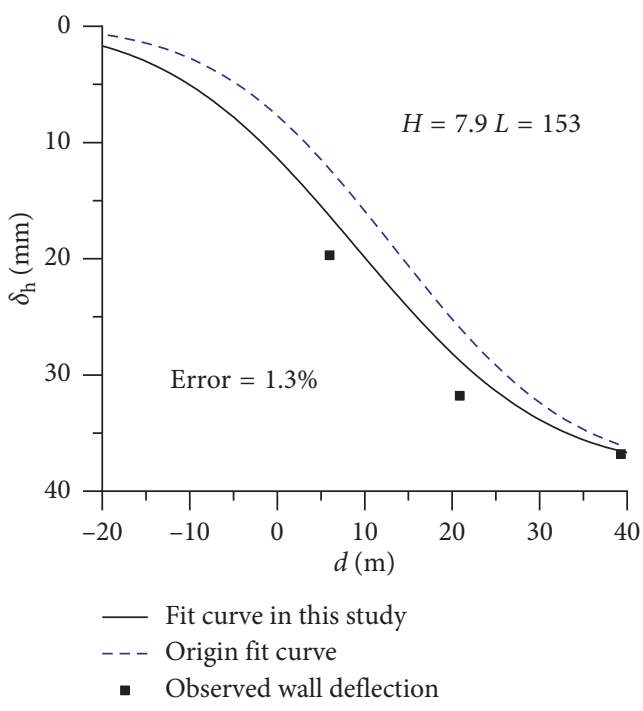

(c)

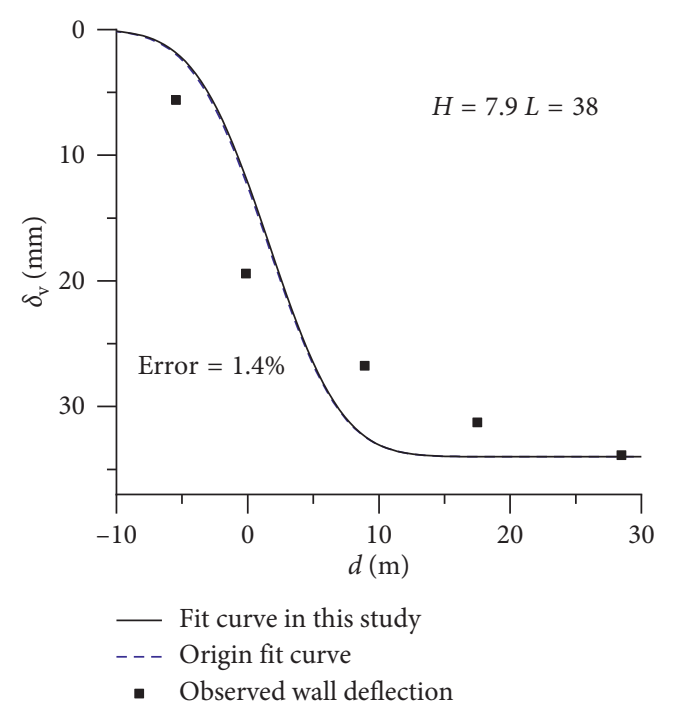

(b)

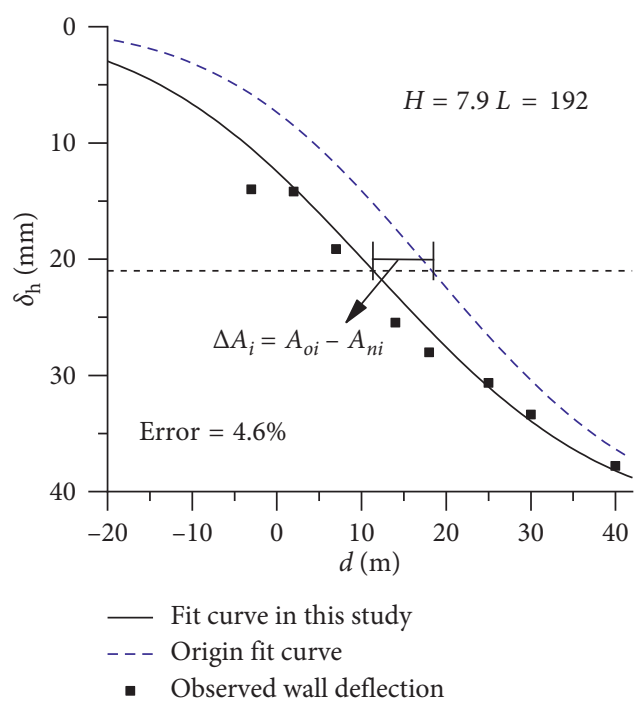

(d)

FIGURE 16: Comparing of the observed and calculated deformation.

This project monitored the settlement of underground pipeline outside the excavation. This paper focused on the D1000 sewage pipeline that is quite close to the wall. The monitoring point arrangement is shown in Figure 4 . The distance between the sewage pipeline and the east wall of Zone $1 \#$ is only $4.3-5.4 \mathrm{~m}$, which is $0.54-0.68 H_{\mathrm{e}}$. It can be seen from Figure 9 that the pipeline is in the primary influence zone outside the excavation.

It was evaluated that the ERFC predicting curve can estimate the ground settlement reasonably. This study attempted to estimate the distribution of surface settlement above the pipeline and compared it with the measured pipeline settlement.

As shown in Figure 17, the location of the point $i$ on the ground that is above the pipeline is determined by using $x_{i}$ (i.e., distance from the corner) and $d_{i}$ (i.e., distance from the retaining wall). Combining the estimation curve of Hsieh and $\mathrm{Ou}$ [25] with the ERFC prediction curve, the settlement of the point $i$ above the pipeline can be estimated by the following equation set:

$$
\left\{\begin{array}{l}
\delta_{\mathrm{vi}}=\delta_{\mathrm{vmi}}\left(\frac{d_{i}}{H_{\mathrm{e}}}+0.5\right), \quad 0 \leq d_{i} / H_{\mathrm{e}}<0.5, \\
\delta_{\mathrm{vi}}=\delta_{\mathrm{vmi}}\left(\frac{-0.6 d_{i}}{H_{\mathrm{e}}}+1.3\right), \quad 0.5 \leq d_{i} / H_{\mathrm{e}} \leq 2.0, \\
\delta_{\mathrm{vmi}}=\delta_{\mathrm{vm}}\left[1-\frac{1}{2} \operatorname{erfc}\left(\frac{2.8\left(x_{i}+0.39 H_{\mathrm{e}}^{2} / L-0.06 L\right)}{0.44 L+0.39 H_{\mathrm{e}}^{2} / L}\right)\right] .
\end{array}\right.
$$

The measured settlement of the pipeline and the calculated final settlement of the ground surface above the pipeline are shown in Figure 18. As can be seen, the trends of the curves are similar: 


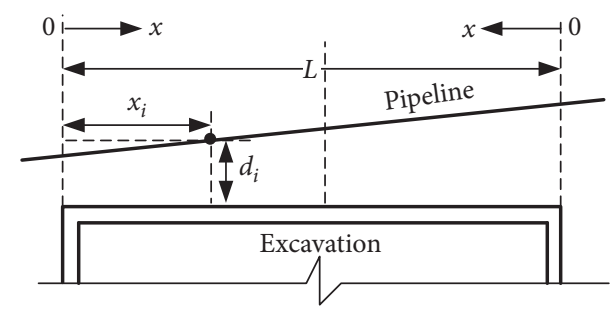

FIgUre 17: Pipeline location schematic diagram.

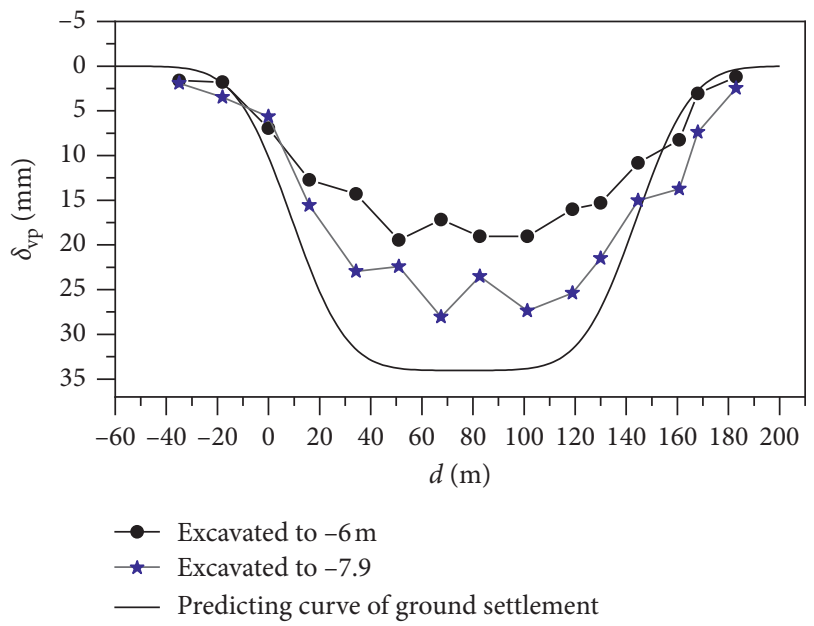

Figure 18: Comparison of the predicted surface settlement and measured pipeline settlement.

$$
\text { disparity }=\frac{\sqrt{(1 / n-1) \sum_{i=1}^{n}\left(\delta_{\mathrm{vpi}}-\delta_{\mathrm{vi}}\right)^{2}}}{\delta_{\mathrm{vpm}}} \times 100 .
$$

The disparity between the measured pipeline settlement and the predicted ground settlement can be determined by using equation (10). The calculated result is $24.7 \%$, and the maximum pipeline settlement is $20 \%$ smaller than the predicted surface settlement. The disparity can be attributed to the bigger rigidity of the pipeline, which may have resisted the pressure of ground settlement. Therefore, when using the ERFC predicting curve to estimate settlement of a reinforced concrete pipeline adjacent to the excavation, a certain reduction can be made and the reduction factor is about 0.8 according to this study. In addition, the maximum distortion of settlement above the pipeline calculated by using equation (6) is about $1 / 1250$, which occurs at a distance of $9 \mathrm{~m}$ from the corner. The maximum distortion of the measured pipeline settlement is $1 / 1600$, which occurs between 0 and $16 \mathrm{~m}$ from the corner. Although the limited quantity of the monitoring point may affect the accuracy of the calculated pipeline maximum distortion, the results suggest that using the ERFC curve to estimate the maximum distortion and the location of the occurrence has certain rationality.

\section{Conclusions}

By analyzing the intensive monitoring data of a long and large excavation (i.e., Lotus port excavation), the influence of the three-dimensional effect on the deformation of the excavation and the adjacent underground pipelines is investigated. And the following conclusions are obtained:

(1) For long and large excavation in soft clay, the maximum wall deflections occurred in a certain range in the middle of the excavation zone, the value is about $0.2 \%-0.6 \% H_{\mathrm{e}}$, fit the range proposed by Kung et al. [3]; the corners have significant inhibition effect on the wall deflections. The inhibition value is about $34 \%-39 \%$ of deflection in the middle range.

(2) Similar to the wall deflections, the ground surface settlements near the corners are significantly inhibited by about $43 \%$ in average; the settlements are in accordance with the distribution curve proposed by Hsieh and $\mathrm{Ou}$ [25].

(3) The partition walls can reduce the nearby wall deflections and ground surface settlements, and the magnitudes of maximum deformations are considerably lower than that in the middle range, even after the partition walls were removed along with the excavation.

(4) The prediction curve proposed by Roboski and Finno [17] has certain deviation for the long and large excavation in this case. A modified curve for estimating the distribution of deformation parallel to the excavation is proposed, and the modified predicting curve fits well the observed data by the error less than $6 \%$. The estimated envelope of $A$ (i.e., the distance from inflection point to corner) is given to predict the location where the maximum distortion will occur, which can help to determine the focus of monitoring and reinforcement outside the excavation.

(5) The modified prediction curve can be used to estimate the pipeline settlement by calculating the ground surface settlement above it, and the reduction coefficient is about 0.8 according to the observed data. The value and the occurrence position of the predicted maximum pipeline distortion can also be reliable reference.

\section{Data Availability}

The data used to support the findings of this study are available from the corresponding author upon request.

\section{Conflicts of Interest}

The authors declare that there are no conflicts of interest regarding the publication of this paper.

\section{Acknowledgments}

The authors would like to thank the National Natural Science Foundation of China (no. 51608485), the 151 Talents Project of Zhejiang Province. 


\section{References}

[1] R. B. Peck, "Deep excavations and tunneling in soft ground," in Proceedings of the Seventh International Conference on Soil Mechanics and Foundation Engineering (ICSMFE), pp. 225290, Mexico, 1969.

[2] G. W. Clough and T. D. O'Rourke, "Construction induced movements of in situ walls," in Proceedings of the ASCE Conference on Design and Performance of Earth Retaining Structures, pp. 439-470, ASCE, New York, NY, USA, June 1990.

[3] G. T. Kung, C. H. Juang, E. C. Hsiao, and Y. M. Hashash, "Simplified model for wall deflection and ground-surface settlement caused by braced excavation in clays," Journal of Geotechnical and Geoenvironmental Engineering, vol. 133, no. 6, pp. 731-747, 2007.

[4] C.-Y. Ou, P.-G. Hsieh, and D.-C. Chiou, "Characteristics of ground surface settlement during excavation," Canadian Geotechnical Journal, vol. 30, no. 5, pp. 758-767, 1993.

[5] C.-Y. Ou, J.-T. Liao, and H.-D. Lin, "Performance of diaphragm wall constructed using top-down method," Journal of Geotechnical and Geoenvironmental Engineering, vol. 124, no. 9, pp. 798-808, 1998.

[6] S. Zhang, G. Ye, C. Liao, and J. Wang, "Elasto-plastic model of structured marine clay under general loading conditions," Applied Ocean Research, vol. 76, pp. 211-220, 2018.

[7] S. Zhang, G. Ye, and J. Wang, "Elasto-plastic model for overconsolidated clays with focus on volume change under general loading conditions," International Journal of Geomechanics, vol. 18, no. 3, article 04018005, 2018.

[8] F.-H. Lee, K.-Y. Yong, K. C. N. Quan, and K.-T. Chee, "Effect of corners in strutted excavations: field monitoring and case histories," Journal of Geotechnical and Geoenvironmental Engineering, vol. 124, no. 4, pp. 339-349, 1998.

[9] C.-Y. Ou, D.-C. Chiou, and T.-S. Wu, "Three-dimensional finite element analysis of deep excavations," Journal of Geotechnical Engineering, vol. 122, no. 5, pp. 337-345, 1996.

[10] C.-Y. Ou, B.-Y. Shiau, and I.-W. Wang, "Three-dimensional deformation behavior of the Taipei National Enterprise Center (TNEC) excavation case history," Canadian Geotechnical Journal, vol. 37, no. 2, pp. 438-448, 2000.

[11] R. J. Finno and L. S. Bryson, "Response of building adjacent to stiff excavation support system in soft clay," Journal of Performance of Constructed Facilities, vol. 16, no. 1, pp. 10-20, 2002.

[12] R. J. Finno and J. F. Roboski, "Three-dimensional responses of a tied-back excavation through clay," Journal of Geotechnical and Geoenvironmental Engineering, vol. 131, no. 3, pp. 273282, 2005.

[13] J. T. Blackburn and R. J. Finno, "Three-dimensional responses observed in an internally braced excavation in soft clay," Journal of Geotechnical and Geoenvironmental Engineering, vol. 133, no. 11, pp. 1364-1373, 2007.

[14] R. J. Finno, J. T. Blackburn, and J. F. Roboski, "Threedimensional effects for supported excavations in clay," Journal of Geotechnical and Geoenvironmental Engineering, vol. 133, no. 1, pp. 30-36, 2007.

[15] J. Shi, C. W. W. Ng, and Y. Chen, "Three-dimensional numerical parametric study of the influence of basement excavation on existing tunnel," Computers and Geotechnics, vol. 63, pp. 146-158, 2015.

[16] C. W. W. Ng, J. Shi, and Y. Hong, "Three-dimensional centrifuge modelling of basement excavation effects on an existing tunnel in dry sand," Canadian Geotechnical Journal, vol. 50, no. 8, pp. 874-888, 2013.
[17] J. Roboski and R. J. Finno, "Distributions of ground movements parallel to deep excavations in clay," Canadian Geotechnical Journal, vol. 43, no. 1, pp. 43-58, 2006.

[18] T. Yong, B. Wei, Y. Diao, and X. Zhou, "Spatial corner effects of long and narrow multipropped deep excavations in Shanghai soft clay," Journal of Performance of Constructed Facilities, vol. 28, no. 4, article 04014015, 2014.

[19] M.-G. Li, J.-J. Chen, J.-H. Wang, and Y.-F. Zhu, "Comparative study of construction methods for deep excavations above shield tunnels," Tunnelling and Underground Space Technology, vol. 71, pp. 329-339, 2018.

[20] M.-G. Li, X. Xiao, J.-H. Wang, and J.-J. Chen, "Numerical study on responses of an existing metro line to staged deep excavations," Tunnelling and Underground Space Technology, vol. 85, pp. 268-281, 2019.

[21] Z. J. Zhang, M. G. Li, J. J. Chen, J. H. Wang, and F. Y. Zeng, "Innovative construction method for oversized excavations with bipartition walls," Journal of Construction Engineering and Management, vol. 143, no. 8, article 04017056, 2017.

[22] P. W. Linehan, A. Longinow, and C. H. Dowding, "Pipeline response to pile driving and adjacent excavation," Journal of Geotechnical Engineering, vol. 118, no. 2, pp. 300-316, 1992.

[23] I. Ahmed, Pipeline Response to Excavation-Induced Ground Movements, Cornell University, Ithaca, NY, USA, 1990.

[24] Y. C. Kog, "Buried pipeline response to braced excavation movements," Journal of Performance of Constructed Facilities, vol. 24, no. 3, pp. 235-241, 2010.

[25] P.-G. Hsieh and C.-Y. Ou, "Shape of ground surface settlement profiles caused by excavation," Canadian Geotechnical Journal, vol. 35, no. 6, pp. 1004-1017, 1998.

[26] Y. Tan and B. Wei, "Observed behaviors of a long and deep excavation constructed by cut-and-cover technique in Shanghai soft clay," Journal of Geotechnical and Geoenvironmental Engineering, vol. 138, no. 1, pp. 69-88, 2012. 


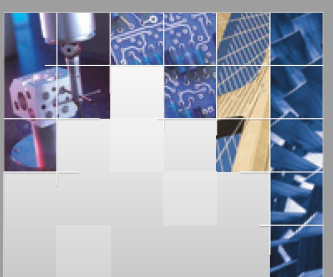

\section{Enfincering}
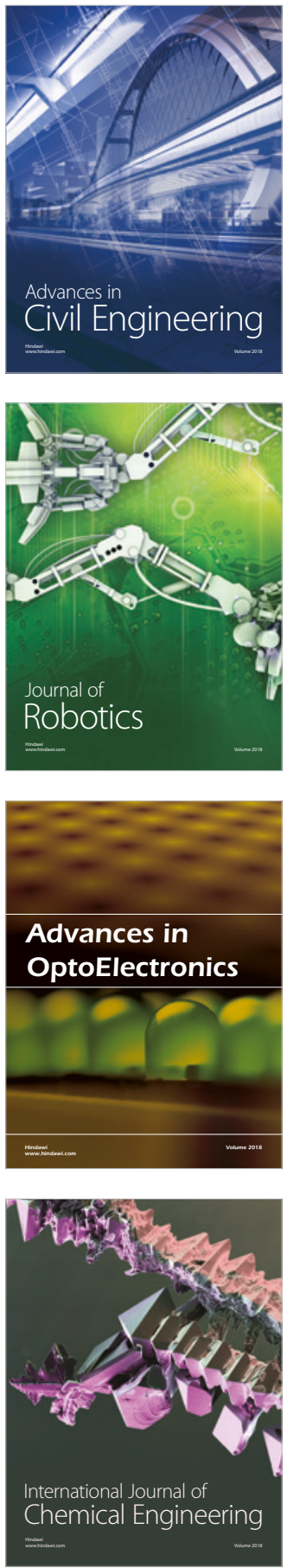

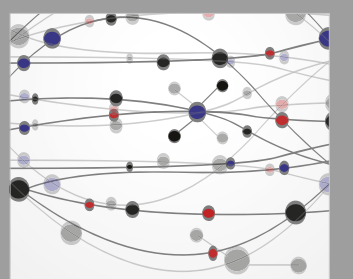

\section{Rotating \\ Machinery}

The Scientific World Journal

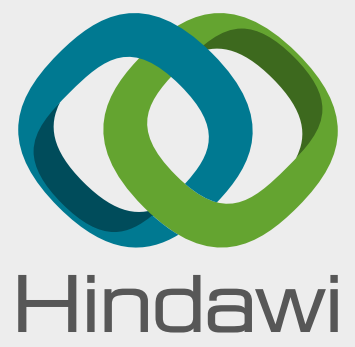

Submit your manuscripts at

www.hindawi.com
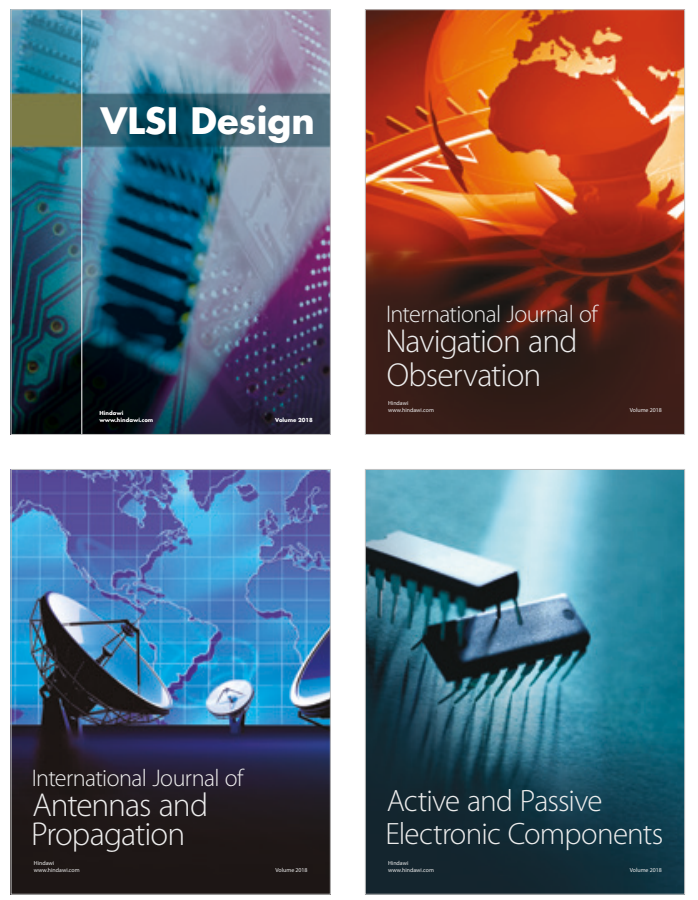
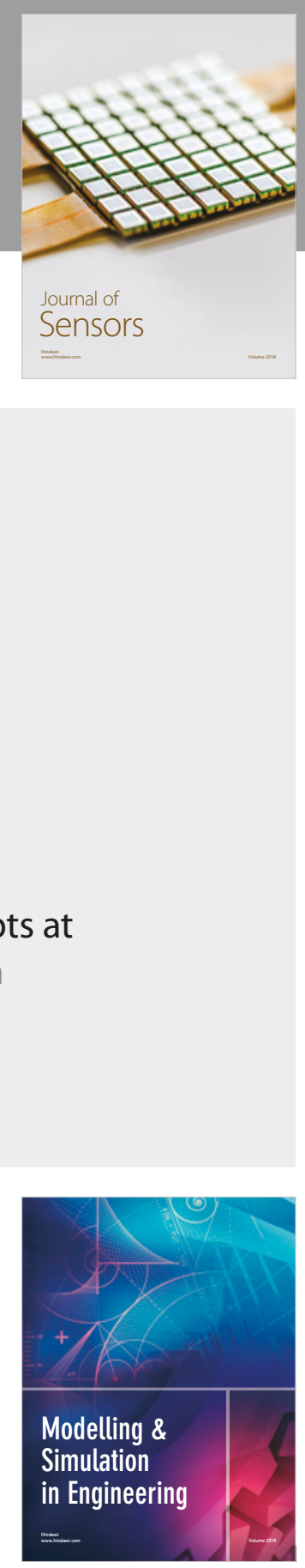

\section{Advances \\ Multimedia}
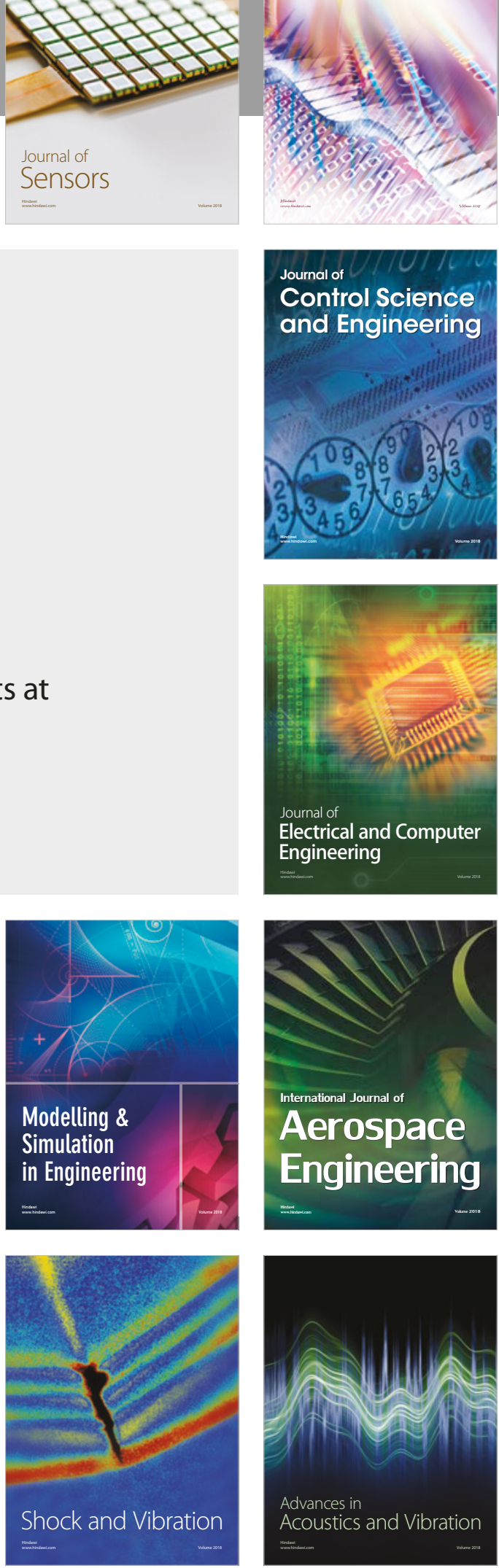